\title{
Comparative effects of angiotensin-converting enzyme inhibitors and angiotensin II receptor blockers on the risk of pneumonia and severe exacerbations in patients with COPD
}

This article was published in the following Dove Press journal:

International Journal of COPD

\author{
Chih-Cheng Lai' \\ Ya-Hui Wang ${ }^{2}$ \\ Cheng-Yi Wang ${ }^{3}$ \\ Hao-Chien Wang ${ }^{4}$ \\ Chong-Jen $\mathrm{Yu}^{4}$ \\ Likwang Chen ${ }^{5}$ \\ On behalf of the Taiwan \\ Clinical Trial Consortium \\ for Respiratory Diseases \\ (TCORE)
}

'Department of Intensive Care Medicine, Chi Mei Medical Center, Liouying, Taiwan; ${ }^{2}$ Medical Research Center, Cardinal Tien Hospital and School of Medicine, College of Medicine, Fu Jen Catholic University, New Taipei City, Taiwan; ${ }^{3}$ Department of Internal Medicine, Cardinal Tien Hospital and School of Medicine, College of Medicine, Fu Jen Catholic University, New Taipei City, Taiwan; ${ }^{4}$ Department of Internal Medicine, National Taiwan University Hospital and College of Medicine, National Taiwan University, Taipei, Taiwan; ${ }^{5}$ Institute of Population Health Sciences, National Health Research Institutes, Zhunan, Taiwan

Correspondence: Cheng-Yi Wang Department of Internal Medicine, Cardinal Tien Hospital and School of Medicine, College of Medicine, Fu Jen Catholic University, Number 362, Zhongzheng Road, Xindian District, New Taipei City 23I48, Taiwan

Tel +886222193391 ext I5433

Fax +886 222219 065 I

Email cywang@mospital.com
Objectives: This study aimed to compare the effects of angiotensin-converting-enzyme inhibitors (ACEis) and angiotensin receptor blockers (ARBs) on the risk of pneumonia and severe exacerbations in patients with COPD.

Patients and methods: All patients with COPD who used ACEis and ARBs for $>90$ days between 2000 and 2005 were recruited. Pairwise matching (1:1) of the ACEi and ARB groups resulted in two similar subgroups, with 6,226 patients in each. The primary outcomes were pneumonia and COPD exacerbations, and the secondary outcome was death.

Results: During the follow-up period, the incidence of pneumonia was 7.20 per 100 personyears in the ACEi group and 5.89 per 100 person-years in the ARB group. The ACEi group had a higher risk of pneumonia (adjusted hazard ratio [aHR], 1.22; 95\% CI, 1.15-1.29) than the ARB group. The incidence of severe exacerbations was 0.65 per person-year for the patients receiving ACEis and 0.52 per person-year for those receiving ARBs. The patients receiving ACEis had a higher risk of severe exacerbations (aHR, 1.19; 95\% CI, 1.16-1.21) than those receiving ARBs. Similar trends were noted in terms of severe exacerbations requiring hospitalization (aHR, 1.24; 95\% CI, 1.21-1.28) or emergency department visits (aHR, 1.16; 95\% CI, 1.13-1.18), pneumonia requiring mechanical ventilation (aHR, 1.35; 95\% CI, 1.24-1.47), and mortality (aHR, 1.33; 95\% CI, 1.26-1.42).

Conclusion: ARBs were associated with lower rates of pneumonia, severe pneumonia, and mortality than ACEis in patients with COPD.

Keywords: angiotensin-converting enzyme inhibitor, angiotensin receptor blocker, COPD, severe exacerbation, pneumonia, mortality

\section{Introduction}

The prevalence of COPD is increasing globally, and COPD has become a major cause of mortality. ${ }^{1,2}$ In patients with COPD, respiratory tract infections such as pneumonia are a common cause of COPD exacerbations, and frequent exacerbations can result in a greater decline in health status. In addition, patients with COPD are at a higher risk of pulmonary infection than the general population. ${ }^{3,4}$ Moreover, many studies ${ }^{5-7}$ have shown that inhaled and systemic corticosteroid therapy, which is frequently used to control COPD and manage COPD exacerbations, can further increase the risk of pneumonia. Therefore, preventing pneumonia in patients with COPD has become an important issue. In addition to vaccination which is recommended by the Global initiative for chronic Obstructive Lung Disease guidelines, ${ }^{8}$ a nested case-control 
study reported that the use of angiotensin-converting enzyme inhibitors (ACEis) and angiotensin receptor blockers (ARBs) was associated with a lower risk of pneumonia in patients with COPD. ${ }^{9}$

Although some studies ${ }^{9-12}$ have investigated the effect of $\mathrm{ACEis}$ and ARBs on the risk of pneumonia, the issue remains controversial due to differences in the study population and effects. One recent meta-analysis ${ }^{10}$ reported that ACEis exhibited a preventive effect equating to a relative risk ranging from 0.32 to 0.81 compared with controls, and that the overall relative risk of ACEi-treated patients versus controls was 0.61 (95\% CI, $0.51-0.75 ; P<0.001)$. Another population-based case-control study ${ }^{11}$ found that a current prescription for ACEis was associated with a reduction in the risk of pneumonia (adjusted odds ratio, 0.75 ; 95\% CI, 0.65-0.86). In contrast, a case-crossover study using the Taiwan Longitudinal Health Insurance Database found that neither the use of nor the cumulative dose of ACEis or ARBs was associated with the risk of pneumonia among the general population in Taiwan. Moreover, it is not known which renin-angiotensin system blocker (an ACEi or $\mathrm{ARB}$ ) is more effective in preventing pneumonia. ${ }^{13-16}$ To date, no study has compared the effect of ACEi and ARB treatment on the risk of pneumonia in patients with COPD. Therefore, we used a national database to investigate the effect of ACEis and ARBs on the risk of pneumonia in patients with COPD. In addition, we compared the effects of ACEis and ARBs on the risk of severe exacerbations of COPD.

\section{Patients and methods}

\section{Study design and patient selection}

The Taiwan National Health Insurance Research Database (NHIRD) is a database constructed by the National Health Research Institutes, and includes the comprehensive medical care records of $>97 \%$ of the hospitals and clinics in Taiwan. We retrieved all claims data of ambulatory care records, outpatient visits, prescriptions, inpatient care records, registration files, and disease and vital status data from the NHIRD. The patient records and information were anonymized and de-identified prior to analysis. The study protocol was approved by the Institutional Review Board of Cardinal Tien Hospital (CTH-104-3-5-030).

This study used a subset of the NHIRD comprising information on individuals with COPD. ${ }^{5,7}$ There were 62,505 eligible COPD patients who were aged older than 40 years between 2000 and 2005. We excluded 40,026 patients who did not meet the following criteria: 1) patients without prescription of ACEi or ARB before COPD index date; 2) patients who received ACEi or ARB $<90$ days after
COPD index date; 3 ) patients with ACEi and ARB combined treatment; and 4) patients who died or were diagnosed with pneumonia or severe pneumonia prior to being indexed (Figure 1). Eligible patients who received prescriptions for an ACEi or ARB within 90 days after diagnosis of COPD were allocated to the ACEi and ARB cohorts. After 1:1 propensity score matching, the remaining 12,452 patients were included for further analysis (Figure 1).

\section{Exposure measures and potential confounders}

We included patients who received ACEis or ARBs for $>90$ days. Therefore, the index date was defined as the date of the 91st day of the prescription of ACEis or ARBs after a diagnosis of COPD. The patients were followed from the index date to 31 December 2011, the end of drug treatment, emigration, or death, whichever occurred first. The patients who changed from an ACEi to an ARB or from an $\mathrm{ARB}$ to an ACEi were censored.

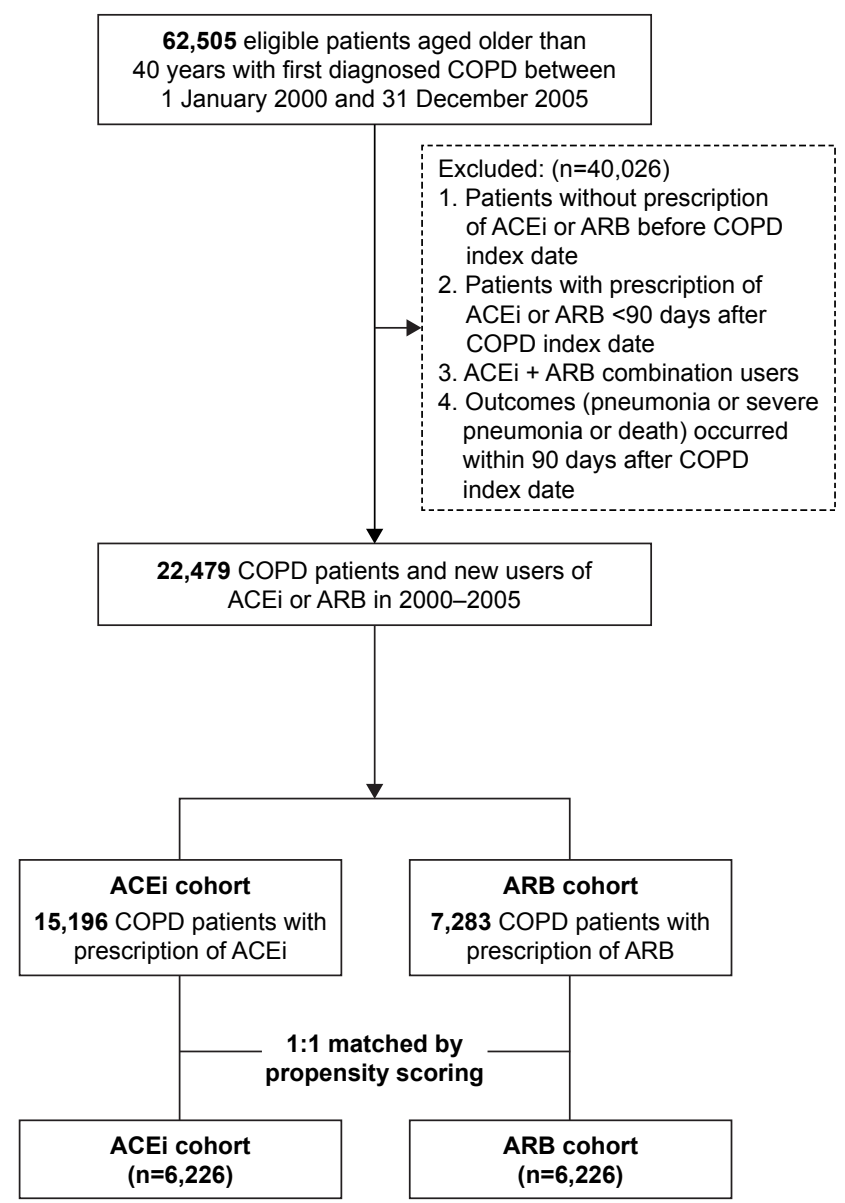

Figure I Flowchart of selection of study subjects.

Abbreviations: ACEi, angiotensin-converting enzyme inhibitor; ARB, angiotensin II receptor blocker. 


\section{Outcome measurements}

The primary outcomes were pneumonia and severe exacerbations. The patients with pneumonia were identified according to the International Classification of Diseases, Ninth Revision, Clinical Modification codes as previously described. ${ }^{5,17}$ For patients with more than one episode of pneumonia, only the first episode was included. A severe exacerbation was defined as a COPD-related hospitalization or emergency department visit. ${ }^{5}$ The first episode of a severe exacerbation and the frequency of severe sepsis (every year) were recorded. The secondary outcome was mortality.

\section{Demographic characteristics and comorbidities}

The demographic characteristics of the patients, including age, gender, monthly income $(<\mathrm{NT} \$ 19,100$, NT\$ 19,100-NT\$ 41,999, and >NT\$ 42,000), hospital level at admission (medical center, regional, district, and others), and severe exacerbations of COPD in the 1 year prior to the index date (never, 1 , or $\geq 2$ times/year), were extracted. Underlying comorbid conditions were identified according to the International Classification of Diseases, Ninth Revision, Clinical Modification codes and grouped as follows: myocardial infarction, congestive heart failure, peripheral vascular disease, cerebrovascular disease, dementia, rheumatologic disease, peptic ulcer disease, hemiplegia or paraplegia, renal disease, diabetes, moderate/severe liver disease, and tumor. The Charlson Comorbidity Index was used to determine the severity of comorbidities for each patient. Important medications including aspirin, clopidogrel, ticlopidine, dipyridamole, nitrates, statins, nonsteroidal anti-inflammatory drugs, anti-hyperglycemic drugs, proton-pump inhibitors, medications for hypertension (including alpha-blockers, beta-blockers, calcium channel blockers, and diuretics), and medications for COPD including long-acting beta agonists, short-acting beta agonists, longacting muscarinic antagonists, and inhaled corticosteroids were recorded.

\section{Statistical analysis}

Data analysis was performed using SAS statistical software version 9.4 (SAS Institute Inc., Cary, NC, USA). The baseline characteristics were presented as descriptive statistics (means, SDs, counts, and percentages). Categorical baseline variables were compared using Pearson's chi-square test, and the independent $t$-test was used to compare continuous baseline variables. To minimize the imbalance of baseline characteristics between the ACEi and ARB groups, we performed 1:1 propensity score matching. Covariates that may have caused interference or bias in the association between exposure and outcomes of interest such as demographic characteristics, comorbidities, and severe exacerbations of COPD in the 1 year prior to the index date (never, 1 , or $\geq 2$ times/year) were included in the propensity matching.

Intention-to-treat analysis was used as the primary analysis in this study because of the more reliable real-world estimates of comparative treatment effectiveness. Both cohorts were followed until the study end according to the original treatment allocation, regardless of adherence to or subsequent withdrawal or deviation from the inclusion criteria. In the as-treated analysis, the patients were censored when they switched from an ACEi to an ARB or from an ARB to an ACEi and when they left the study because of death or emigration. We used the last time point that they were known to be alive to censor those without an event. Cumulative incidence curves were constructed using the Kaplan-Meier method, and differences between the two treatment groups were tested using the log-rank test.

The crude incidence rates of individual outcomes were calculated as the total number of events during the follow-up period divided by person-years. Cox proportional regression models were used to calculate crude hazard ratios (HRs) and adjusted hazard ratios (aHRs), which were adjusted for propensity score, of different outcomes between the ACEi and ARB groups. Annual severe exacerbation event rates (emergency department visits or admissions to hospital) were compared between the two groups using Poisson regression, with events as the dependent variable and time on a specific fixed combination treatment as an offset variable. A twosided $P$-value $<0.05$ was considered to indicate statistical significance in all analyses.

\section{Results \\ Characteristics of the study population}

Initially, 22,479 patients with COPD who used ACEis $(n=15,196)$ or ARBs $(n=7,283)$ between 2000 and 2005 were included. There were significant differences between the two groups in some demographic characteristics including index year; age; gender; monthly income; hospital level; episodes of prior severe exacerbations; underlying comorbidities such as myocardial infarction, congestive heart failure, peptic ulcer disease, hemiplegia or paraplegia, and diabetes mellitus; and the use of medications including beta-blockers, calcium channel blockers, diuretics, clopidogrel, ticlopidine, dipyridamole, statins, nonsteroidal anti-inflammatory drugs, and inhalation therapy for COPD (Table 1). Therefore, we used 
Table I Demographic characteristics of the study subjects

\begin{tabular}{|c|c|c|c|c|c|c|c|c|c|c|}
\hline \multirow[t]{2}{*}{ Variables } & \multicolumn{5}{|c|}{ Before matching } & \multicolumn{5}{|c|}{ After matching } \\
\hline & \multicolumn{2}{|c|}{ ACEi cohort } & \multicolumn{2}{|c|}{ ARB cohort } & $P$-value & \multicolumn{2}{|c|}{ ACEi cohort } & \multicolumn{2}{|c|}{ ARB cohort } & $P$-value \\
\hline Number of patients & \multicolumn{2}{|l|}{15,196} & \multicolumn{2}{|l|}{7,283} & & \multicolumn{2}{|l|}{6,226} & \multicolumn{2}{|l|}{6,226} & \\
\hline \multicolumn{11}{|l|}{ Index year } \\
\hline 2000 & 3,763 & $24.76 \%$ & 768 & $10.55 \%$ & $<0.0001$ & 736 & $11.82 \%$ & 768 & $12.34 \%$ & 0.9693 \\
\hline 2001 & 2,985 & $19.64 \%$ & 884 & $12.14 \%$ & & 883 & $14.18 \%$ & 880 & $14.13 \%$ & \\
\hline 2002 & 2,552 & $16.79 \%$ & 1,109 & $15.23 \%$ & & 1,068 & $17.15 \%$ & 1,047 & $16.82 \%$ & \\
\hline 2003 & 2,017 & $13.27 \%$ & 1,336 & $18.34 \%$ & & 1,132 & $18.18 \%$ & 1,130 & $18.15 \%$ & \\
\hline 2004 & 2,002 & $13.17 \%$ & 1,695 & $23.27 \%$ & & $\mathrm{I}, 264$ & $20.30 \%$ & $\mathrm{I}, 264$ & $20.30 \%$ & \\
\hline 2005 & $\mathrm{I}, 877$ & $12.35 \%$ & $|, 49|$ & $20.47 \%$ & & $\mathrm{I}, 143$ & $18.36 \%$ & 1,137 & $18.26 \%$ & \\
\hline Age (years, SD) & \multicolumn{2}{|c|}{$68.15 \pm 9.77$} & \multicolumn{2}{|c|}{$66.75 \pm 9.95$} & $<0.0001$ & \multicolumn{2}{|c|}{$67.43 \pm 10.01$} & \multicolumn{2}{|c|}{$67.24 \pm 9.82$} & 0.2919 \\
\hline Male gender & $|I, 07|$ & $72.85 \%$ & 4,983 & $68.42 \%$ & $<0.0001$ & 4,405 & $70.75 \%$ & 4,359 & $70.01 \%$ & 0.3666 \\
\hline \multicolumn{11}{|l|}{ Monthly income NT\$, n (\%) } \\
\hline$<19,100$ & 6,597 & $43.41 \%$ & 2,973 & $40.82 \%$ & $<0.0001$ & 2,658 & $42.69 \%$ & 2,625 & $42.16 \%$ & 0.8336 \\
\hline $19,100-41,999$ & 7,050 & $46.39 \%$ & 3,262 & $44.79 \%$ & & 2,784 & $44.72 \%$ & 2,807 & $45.09 \%$ & \\
\hline$\geq 42,000$ & 1,549 & $10.19 \%$ & $\mathrm{I}, 048$ & $14.39 \%$ & & 784 & $12.59 \%$ & 794 & $12.75 \%$ & \\
\hline \multicolumn{11}{|l|}{ Hospital level, n (\%) } \\
\hline Level I & 3,360 & $22.11 \%$ & 3,095 & $42.50 \%$ & $<0.0001$ & 2,374 & $38.13 \%$ & 2,366 & $38.00 \%$ & 0.593 \\
\hline Level 2 & 3,625 & $23.85 \%$ & 2,254 & $30.95 \%$ & & 1,959 & $31.46 \%$ & 1,994 & $32.03 \%$ & \\
\hline Level 3 & 3,088 & $20.32 \%$ & 1,335 & $18.33 \%$ & & 1,319 & $21.19 \%$ & 1,267 & $20.35 \%$ & \\
\hline Level 4 & 5,123 & $33.71 \%$ & 599 & $8.22 \%$ & & 574 & $9.22 \%$ & 599 & $9.62 \%$ & \\
\hline \multicolumn{11}{|c|}{ Severe AE (count I year before index date) } \\
\hline 0 & 10,277 & $67.63 \%$ & 5,354 & $73.51 \%$ & $<0.0001$ & 4,466 & $71.73 \%$ & 4,446 & $71.41 \%$ & 0.9181 \\
\hline 1 & 2,190 & $14.4 \mid \%$ & 918 & $12.60 \%$ & & 827 & $13.28 \%$ & 833 & $13.38 \%$ & \\
\hline $2+$ & 2,729 & $17.96 \%$ & $\mathrm{I}, 01 \mathrm{I}$ & $13.88 \%$ & & 933 & $14.99 \%$ & 947 & $15.21 \%$ & \\
\hline \multicolumn{11}{|l|}{ Baseline comorbidities } \\
\hline Charlson score & & 1.06 & & \pm 1.05 & 0.4771 & & \pm 1.07 & & \pm 1.05 & 0.3405 \\
\hline Myocardial infarction & 391 & $2.57 \%$ & 131 & $1.80 \%$ & 0.0003 & 142 & $2.28 \%$ & 128 & $2.06 \%$ & 0.389 \\
\hline Congestive heart failure & 2,164 & $14.24 \%$ & 849 & $11.66 \%$ & $<0.0001$ & 824 & $13.23 \%$ & 796 & $12.79 \%$ & 0.4557 \\
\hline Peripheral vascular disease & 156 & $1.03 \%$ & 71 & $0.97 \%$ & 0.7167 & 70 & $1.12 \%$ & 59 & $0.95 \%$ & 0.3303 \\
\hline Cerebrovascular disease & 1,675 & $11.02 \%$ & 786 & $10.79 \%$ & 0.6047 & 687 & $11.03 \%$ & 679 & $10.91 \%$ & 0.8186 \\
\hline Dementia & 181 & $1.19 \%$ & 87 & $1.19 \%$ & 0.9822 & 81 & $1.30 \%$ & 75 & $1.20 \%$ & 0.6288 \\
\hline Rheumatologic disease & $|4|$ & $0.93 \%$ & 74 & $1.02 \%$ & 0.5249 & 60 & $0.96 \%$ & 66 & $1.06 \%$ & 0.5911 \\
\hline Peptic ulcer disease & 2,830 & $18.62 \%$ & 1,187 & $16.30 \%$ & $<0.0001$ & 1,087 & $17.46 \%$ & 1,068 & $17.15 \%$ & 0.6526 \\
\hline Hemiplegia or paraplegia & 23 & $0.15 \%$ & 15 & $0.21 \%$ & 0.3510 & 9 & $0.14 \%$ & 10 & $0.16 \%$ & 0.8184 \\
\hline Renal disease & 458 & $3.01 \%$ & 275 & $3.78 \%$ & 0.0026 & 216 & $3.47 \%$ & 228 & $3.66 \%$ & 0.562 \\
\hline Diabetes & 1,650 & $10.86 \%$ & 933 & $12.81 \%$ & $<0.0001$ & 799 & $12.83 \%$ & 787 & $12.64 \%$ & 0.747 \\
\hline $\begin{array}{l}\text { Moderate or severe liver } \\
\text { disease }\end{array}$ & 682 & $4.49 \%$ & 337 & $4.63 \%$ & 0.6387 & 281 & $4.51 \%$ & 299 & $4.80 \%$ & 0.444 \\
\hline Tumor & 410 & $2.70 \%$ & 223 & $3.06 \%$ & 0.1228 & 202 & $3.24 \%$ & 191 & $3.07 \%$ & 0.5729 \\
\hline Medication for hypertension & & & & & & & & & & \\
\hline Alpha-blocker & 2,056 & $13.53 \%$ & 936 & $12.85 \%$ & 0.1613 & 846 & $13.59 \%$ & 831 & $13.35 \%$ & 0.6938 \\
\hline Beta-blocker & 6,354 & $41.81 \%$ & 3,156 & $43.33 \%$ & 0.0309 & $2,67 I$ & $42.90 \%$ & 2,671 & $42.90 \%$ & I \\
\hline Calcium channel blocker & 9,748 & $64.15 \%$ & 5,128 & $70.41 \%$ & $<0.0001$ & 4,299 & $69.05 \%$ & 4,306 & $69.16 \%$ & 0.892 \\
\hline Diuretic & 7,825 & $51.49 \%$ & 3,474 & $47.70 \%$ & $<0.0001$ & 3,095 & $49.71 \%$ & 3,042 & $48.86 \%$ & 0.3421 \\
\hline Other medication & & & & & & & & & & \\
\hline Aspirin & 2,220 & $14.61 \%$ & 1,115 & $15.31 \%$ & 0.1667 & 953 & $15.31 \%$ & 927 & $14.89 \%$ & 0.5152 \\
\hline Clopidogrel & 356 & $2.34 \%$ & 340 & $4.67 \%$ & $<0.0001$ & 265 & $4.26 \%$ & 267 & $4.29 \%$ & 0.9294 \\
\hline
\end{tabular}


Table I (Continued)

\begin{tabular}{|c|c|c|c|c|c|c|c|c|c|c|}
\hline \multirow{3}{*}{$\begin{array}{l}\text { Variables } \\
\text { Ticlopidine }\end{array}$} & \multicolumn{5}{|c|}{ Before matching } & \multicolumn{5}{|c|}{ After matching } \\
\hline & \multicolumn{2}{|c|}{ ACEi cohort } & \multicolumn{2}{|c|}{ ARB cohort } & \multirow{2}{*}{$\frac{P \text {-value }}{0.0180}$} & \multicolumn{2}{|c|}{ ACEi cohort } & \multicolumn{2}{|c|}{ ARB cohort } & \multirow{2}{*}{$\frac{P \text {-value }}{0.9612}$} \\
\hline & 591 & $3.89 \%$ & 237 & $3.25 \%$ & & 219 & $3.52 \%$ & 220 & $3.53 \%$ & \\
\hline Dipyridamole & 4,415 & $29.05 \%$ & $\mathrm{I}, 563$ & $21.46 \%$ & $<0.0001$ & $\mathrm{I}, 444$ & $23.19 \%$ & 1,432 & $23.00 \%$ & 0.7986 \\
\hline Nitrate & 46 & $0.30 \%$ & 23 & $0.32 \%$ & 0.8681 & 21 & $0.34 \%$ & 22 & $0.35 \%$ & 0.8786 \\
\hline Statin & 1,167 & $7.68 \%$ & 1,031 & $14.16 \%$ & $<0.0001$ & 725 & $11.64 \%$ & 733 & $11.77 \%$ & 0.8236 \\
\hline NSAID & 12,905 & $84.92 \%$ & 5,929 & $81.41 \%$ & $<0.0001$ & 5,073 & $81.48 \%$ & 5,111 & $82.09 \%$ & 0.3776 \\
\hline Anti-hyperglycemic drugs & 2,560 & $16.85 \%$ & 1,275 & $17.51 \%$ & 0.2183 & 1,127 & $18.10 \%$ & 1,102 & $17.70 \%$ & 0.5589 \\
\hline Proton-pump inhibitor & $|, 21|$ & $7.97 \%$ & 611 & $8.39 \%$ & 0.2800 & 547 & $8.79 \%$ & 533 & $8.56 \%$ & 0.6558 \\
\hline \multicolumn{11}{|l|}{ COPD drug } \\
\hline Long-acting beta-agonist & 670 & $4.41 \%$ & 396 & $5.44 \%$ & 0.0007 & 317 & $5.09 \%$ & 327 & $5.25 \%$ & 0.6857 \\
\hline Short-acting beta-agonist & 3,171 & $20.87 \%$ & 1,432 & $19.66 \%$ & $0.036 I$ & 1,253 & $20.13 \%$ & 1,236 & $19.85 \%$ & 0.7032 \\
\hline $\begin{array}{l}\text { Long-acting muscarinic } \\
\text { antagonist }\end{array}$ & 85 & $0.56 \%$ & 80 & $1.10 \%$ & $<0.0001$ & 59 & $0.95 \%$ & 60 & $0.96 \%$ & 0.9266 \\
\hline Inhaled corticosteroid & 2,323 & $15.29 \%$ & $\mathrm{I}, 479$ & $20.31 \%$ & $<0.0001$ & $\mathrm{I}, \mathrm{I} 47$ & $18.42 \%$ & 1,196 & $19.21 \%$ & 0.2612 \\
\hline
\end{tabular}

Abbreviations: AE, acute exacerbation; ACEi, angiotensin-converting enzyme inhibitor; ARB, angiotensin II receptor blocker; NSAID, nonsteroidal anti-inflammatory drug.

pairwise matching (1:1) of the ACEi and ARB groups, which resulted in two similar subgroups with 6,226 patients in each. There were no significant differences in index year, age, sex, monthly income, hospital level, inhaled medications, antihypertension medications, or commonly used medications including aspirin, clopidogrel, ticlopidine, dipyridamole, anti-hyperglycemic agents, and proton-pump inhibitors (Table 1).

\section{Effect on the risk of severe exacerbations}

Following matching, the post-index all severe exacerbation rates were 0.6521 and 0.5198 per person-year in the ACEi and ARB groups, respectively (adjusted rate ratio [RR], 1.19; 95\% CI, 1.16-1.21), as shown in Table 2. The patients treated with ACEis had significantly higher rates of COPD exacerbations requiring hospitalization (adjusted RR, 1.24; 95\% CI, 1.21-1.28) or emergency department visit (adjusted RR, 1.16; 95\% CI, 1.13-1.18), as shown in Table 2. The results before matching were the same as after matching.

\section{Effect on the risk of pneumonia and death}

During the follow-up period, the incidence of pneumonia was 7.39 per 100 person-years for the patients receiving ACEis and 5.66 per 100 person-years for those receiving ARBs. Moreover, the ACEi group had a higher risk of pneumonia (aHR, 1.20; 95\% CI, 1.14-1.26) than the ARB group. Furthermore, the incidence of pneumonia requiring mechanical ventilation was 2.90 per 100 person-years in the ACEi group and 1.97 per 100 person-years in the ARB group, and the ACEi group had a higher risk of pneumonia requiring mechanical ventilation (aHR, 1.34; 95\% CI, 1.24-1.45) than the ARB group. Finally, the mortality rate was 5.82 per 100 person-years in the ACEi group and 3.90 per 100 person-years in the ARB group. Overall, the patients

Table 2 Annual incidence and RR of severe exacerbation of COPD

\begin{tabular}{|c|c|c|c|c|}
\hline \multirow[t]{2}{*}{ Variables } & \multirow{2}{*}{$\begin{array}{l}\text { ACEi cohort } \\
\text { Annual incidence } \\
\text { (per person-year) }\end{array}$} & \multirow{2}{*}{$\begin{array}{l}\text { ARB cohort } \\
\text { Annual incidence } \\
\text { (per person-year) }\end{array}$} & \multirow{2}{*}{$\begin{array}{l}\text { Crude RR } \\
(95 \% \mathrm{CI})\end{array}$} & \multirow{2}{*}{$\begin{array}{l}\text { Adjusted } \mathbf{R R}^{\mathrm{a}} \\
(95 \% \mathrm{CI})\end{array}$} \\
\hline & & & & \\
\hline \multicolumn{5}{|l|}{ Before propensity score matching } \\
\hline Severe exacerbation & 0.6873 & 0.5109 & $1.32(1.30-1.34)$ & $1.19(1.17-1.21)$ \\
\hline Hospitalization for COPD exacerbation & 0.3573 & 0.23579 & $1.49(1.46-1.52)$ & $1.27(1.25-1.30)$ \\
\hline Emergency department visits for COPD exacerbation & 0.4639 & 0.3765 & $1.21(1.19-1.23)$ & $1.14(1.12-1.16)$ \\
\hline \multicolumn{5}{|l|}{ After propensity score matching } \\
\hline Severe exacerbation & 0.6521 & 0.5198 & $1.19(1.17-1.21)$ & $1.19(1.16-1.21)$ \\
\hline Hospitalization for COPD exacerbation & 0.3213 & 0.2438 & $1.25(1.21-1.28)$ & $1.24(1.2 \mid-1.28)$ \\
\hline Emergency department visits for COPD exacerbation & 0.4620 & 0.3778 & $1.16(1.13-1.18)$ & $1.16(1.13-1.18)$ \\
\hline
\end{tabular}

Note: ${ }^{a}$ Adjusted for propensity score.

Abbreviations: ACEi, angiotensin-converting enzyme inhibitor; ARB, angiotensin II receptor blocker; RR, rate ratio. 
Table 3 Annual IR and risk of pneumonia among COPD patients

\begin{tabular}{|c|c|c|c|c|c|c|c|c|c|}
\hline \multirow[t]{2}{*}{ Variables } & \multicolumn{3}{|c|}{ ACEi cohort } & \multicolumn{3}{|c|}{ ARB cohort } & \multirow{2}{*}{$\frac{\text { Crude }}{\text { HR (95\% Cl) }}$} & \multirow{2}{*}{$\frac{\text { Adjusted }^{\mathrm{b}}}{\text { HR }(95 \% \mathrm{Cl})}$} & \multirow{2}{*}{$\frac{\text { Competing risk }}{\text { subHR }(95 \% \mathrm{Cl})}$} \\
\hline & Event & Person-year & $\mathbf{I R}^{\mathbf{a}}$ & Event & Person-year & $\mathbf{I R}^{\mathbf{a}}$ & & & \\
\hline \multicolumn{10}{|c|}{ Before propensity score matching } \\
\hline Mortality & 6,277 & $107,841.55$ & 5.82 & 2,057 & $52,759.75$ & 3.90 & $1.50(1.42-1.57)$ & $1.29(1.22-1.37)$ & - \\
\hline Pneumonia & 6,516 & $88,|35.3|$ & 7.39 & 2,564 & $45,316.88$ & 5.66 & $1.30(1.24-1.36)$ & $1.20(1.14-1.26)$ & $1.15(1.09-1.20)$ \\
\hline Pneumonia requiring $\mathrm{MV}$ & 3,005 & $103,492.68$ & 2.90 & 1,010 & $51,298.54$ & 1.97 & $1.47(1.37-1.58)$ & $1.34(1.24-1.45)$ & $1.15(1.09-1.20)$ \\
\hline \multicolumn{10}{|c|}{ After propensity score matching } \\
\hline Mortality & 2,326 & $42,503.29$ & 5.47 & $\mathrm{I}, 864$ & $45,472.26$ & 4.10 & $1.33(1.25-1.42)$ & $1.33(1.26-1.42)$ & - \\
\hline Pneumonia & 2,536 & $35,204.12$ & 7.20 & 2,281 & $38,750.59$ & 5.89 & $1.22(1.15-1.29)$ & $1.22(1.15-1.29)$ & $1.19(1.13-1.26)$ \\
\hline Pneumonia requiring $\mathrm{MV}$ & 1,122 & $40,864.53$ & 2.75 & 899 & $44,158.63$ & 2.04 & $1.35(1.23-1.47)$ & I.35 (I.24-I.47) & $1.19(1.13-1.25)$ \\
\hline
\end{tabular}

Notes: aPer 100 person-years. ${ }^{b}$ Adjusted for propensity score.

Abbreviations: ACEi, angiotensin-converting enzyme inhibitor; ARB, angiotensin II receptor blocker; HR, hazard ratio; IR, incidence rate; MV, mechanical ventilation; subHR, subdistribution hazard ratio.

receiving ACEis had a higher risk of death (aHR, 1.29; 95\% CI, 1.22-1.37) than those receiving ARBs. When we treated death as a competing risk, the ACEi group had a higher risk of pneumonia and pneumonia requiring mechanical ventilation than the ARB group. Similar trends were noted after using propensity score matching, and the ACEi group had a higher risk of pneumonia and pneumonia requiring mechanical ventilation than the ARB group, even when treating death as a competing risk (Table 3 ).

\section{Sensitivity analysis}

Table 4 shows the sensitivity analysis of the risk of pneumonia and death among the patients with COPD receiving ACEis compared to the matched patients receiving ARBs. When we treated death as a competing risk in the intentionto-treat analysis, the patients receiving ACEis had a higher risk of pneumonia and pneumonia requiring mechanical ventilation compared to the matched patients receiving ARBs (aHR, 1.19; 95\% CI, 1.13-1.26 for pneumonia; aHR, 1.19; 95\% CI, 1.13-1.25 for pneumonia requiring mechanical ventilation). In the as-treated analysis, the patients receiving ACEis had a higher risk of pneumonia and pneumonia requiring mechanical ventilation compared to the matched patients receiving ARBs. When death was treated as a competing risk in the as-treated analysis, the patients receiving ACEis had a higher risk of pneumonia than those receiving ARBs (aHR, $1.08 ; 95 \% \mathrm{CI}, 1.01-1.15)$. The results of analysis conducted before propensity score matching were consistent with those after propensity score matching.

\section{Discussion}

In this large national study, we compared the effect of the prior use of ACEis and ARBs on the risk of pneumonia and severe exacerbations in patients with COPD. After adjusting

Table 4 Sensitivity analysis

\begin{tabular}{|c|c|c|c|c|}
\hline \multirow[t]{3}{*}{ Variables } & \multicolumn{2}{|c|}{ Before matching } & \multicolumn{2}{|l|}{ After matching } \\
\hline & Crude & Adjusted ${ }^{\mathrm{a}}$ & Crude & Adjusted $^{\mathrm{a}}$ \\
\hline & HR (95\% CI) & HR (95\% CI) & HR (95\% CI) & HR (95\% CI) \\
\hline \multicolumn{5}{|l|}{ Primary analysis } \\
\hline Mortality & I.5 (I.42-I.57) & $1.29(1.22-1.37)$ & $\mathrm{I} .33(\mathrm{I} .25-\mathrm{I} .42)$ & $1.33(1.26-1.42)$ \\
\hline Pneumonia & $\mathrm{I} .3(\mathrm{I} .24-\mathrm{I} .36)$ & $\mathrm{I} .2(\mathrm{I} . \mathrm{I} 4-\mathrm{I} .26)$ & $1.22(1.15-1.29)$ & $1.22(1.15-1.29)$ \\
\hline Pneumonia requiring MV & $1.47(1.37-1.58)$ & $1.34(1.24-1.45)$ & $1.35(1.23-1.47)$ & $1.35(1.24-1.47)$ \\
\hline \multicolumn{5}{|l|}{ ITT analysis + competing risk } \\
\hline \multicolumn{5}{|l|}{ Mortality } \\
\hline Pneumonia & $\mathrm{I} .04(\mathrm{I}-\mathrm{I} .09)$ & $1.15(1.09-1.2)$ & $1.19(1.13-1.26)$ & $1.19(1.13-1.26)$ \\
\hline Pneumonia requiring $\mathrm{MV}$ & $0.96(0.92-1)$ & I.I5 (I.09-I.2) & $1.19(1.12-1.25)$ & $1.19(1.13-1.25)$ \\
\hline \multicolumn{5}{|l|}{ As-treated analysis } \\
\hline Mortality & $1.5(I .4-1.6 I)$ & $1.28(1.19-1.38)$ & $1.29(1.19-1.4)$ & I.3 (I.2-I.4I) \\
\hline Pneumonia & $1.28(1.21-1.36)$ & $1.17(1.1-1.26)$ & $1.16(1.08-1.25)$ & $1.17(1.09-1.26)$ \\
\hline Pneumonia requiring $M V$ & $1.38(1.25-1.52)$ & $1.25(1.13-1.39)$ & I.2I (I.07-I.36) & I.2I (I.08-I.37) \\
\hline \multicolumn{5}{|c|}{ As-treated analysis + competing risk } \\
\hline \multicolumn{5}{|l|}{ Mortality } \\
\hline Pneumonia & $1.07(1.01-1.13)$ & $1.06(I-I .12)$ & I.07 (I-I.I5) & $1.08(1.01-1.15)$ \\
\hline Pneumonia requiring $\mathrm{MV}$ & 1.01 (0.96-1.07) & I.03 (0.97-I.I) & $1.04(0.98-1.12)$ & $\mathrm{I} .04(0.97-\mathrm{I} .12)$ \\
\hline
\end{tabular}

Note: ${ }^{a}$ Adjusted for propensity score.

Abbreviations: HR, hazard ratio; ITT, intention-to-treat; MV, mechanical ventilation. 
for possible confounding factors and using different methods of analysis, the risks of pneumonia and severe exacerbations were significantly higher in the patients who received ACEis than in those who received ARBs. In addition, the patients with COPD who used ACEis had a significantly higher mortality rate than those who used ARBs. Taken together, these findings suggest that ARBs are associated with fewer COPD complications, including severe exacerbations, pneumonia, and mortality, than ACEis, and this may further suggest that ARBs are more appropriate for patients with COPD, in terms of the risk of severe exacerbations and pneumonia, than ACEis.

A previous study investigating the effects of ACEis and ARBs on the risk of pneumonia did not find any obvious differences between ACEis and ARBs in the general population. ${ }^{12}$ In contrast, another study enrolling patients with hypertension found that ARB users, but not ACEi users had lower rates of hospitalization for sepsis, compared with untreated hypertensive patients. ${ }^{13}$ In this study, we found significant differences between ACEi and ARB treatments in terms of COPD exacerbations and the risk of pneumonia. The difference between this study and previous reports ${ }^{12,13}$ may be due to different study populations, and further studies are warranted to investigate differences in the effects between ACEis and ARBs in specific populations. Regarding the effect on mortality between the ACEi and ARB groups, our findings are consistent with a time-matched nested case-control study in which ARB users seemed to have a lower mortality rate than ACEi users. ${ }^{6}$

The significant difference in complications of COPD between users of ARBs and ACEis may be explained by their different mechanisms of action on the renin-angiotensin system in pulmonary diseases. ${ }^{10,11,18}$ In an animal model, valsartan was shown to be protective against bleomycininduced pulmonary injuries via the suppression of total and active transforming growth factor-beta $1 .{ }^{11}$ Another investigation ${ }^{18}$ demonstrated that candesartan can reduce bleomycin-induced lung fibrosis. In addition, ARBs have been reported to be able to use the ACE2/AT2R/Mas axis to ameliorate inflammation. ${ }^{19,20}$ These additional effects of ARBs may result in the differences with ACEis with regards to the risk and outcomes of severe exacerbations and pneumonia in patients with COPD.

The strength of this study is that we enrolled a large cohort of patients with COPD and various cardiovascular risk profiles with a long follow-up period, which represents the general condition in real-word practice. Based on the data from the NHIRD, we were able to analyze many known confounding factors including comorbidities, drug usage, and the frequency of severe exacerbations, which allowed us to minimize the effects of confounders.

This study also has several limitations. First, as with all studies based on claims databases, some confounders such as pulmonary function and clinical symptoms and signs were not available for analysis. Therefore, we could not evaluate the severity of COPD. However, we used episodes of severe exacerbations to partly represent the severity of COPD. Second, only data on all-cause mortality are contained in the NHIRD, so we could not analyze specific causes of death. Therefore, we could not analyze whether the difference in mortality rates between the ACEi and ARB groups was due to cardiovascular event-related death or another etiology.

\section{Conclusion}

ARB treatment was associated with lower rates of severe exacerbations, pneumonia, and mortality than ACEi treatment in patients with COPD.

\section{Acknowledgments}

This work was supported by grants from the National Science Council (NSC 104-2314-B-002-185-MY2, 101-2325-B-002064, 102-2325-B-002-087, 103-2325-B-002-027, 104-2325B-002-035, 105-2325-B-002-030, 104-2314-B-567-002, and 105-2314-B-567-001) and from the National Health Research Institutes (intramural funding).

\section{Disclosure}

The authors report no conflicts of interest in this work.

\section{References}

1. Lozano R, Naghavi M, Foreman K, et al. Global and regional mortality from 235 causes of death for 20 age groups in 1990 and 2010: a systematic analysis for the Global Burden of Disease Study 2010. Lancet. 2012;380(9859):2095-2128.

2. Mathers CD, Loncar D. Projections of global mortality and burden of disease from 2002 to 2030. PLoS Med. 2006;3(11):e442.

3. Lange P. Chronic obstructive pulmonary disease and risk of infection. Pneumonol Alergol Pol. 2009;77(3):284-288.

4. Lv Y, Lv Q, Lv Q, Lai T. Pulmonary infection control window as a switching point for sequential ventilation in the treatment of COPD patients: a meta-analysis. Int J Chron Obstruct Pulmon Dis. 2017;12: 1255-1267.

5. Yang HH, Lai CC, Wang YH, et al. Severe exacerbation and pneumonia in COPD patients treated with fixed combinations of inhaled corticosteroid and long-acting beta2 agonist. Int J Chron Obstruct Pulmon Dis. 2017 12:2477-2485.

6. Mancini GB, Etminan M, Zhang B, Levesque LE, FitzGerald JM, Brophy JM. Reduction of morbidity and mortality by statins, angiotensinconverting enzyme inhibitors, and angiotensin receptor blockers in patients with chronic obstructive pulmonary disease. J Am Coll Cardiol. 2006;47(12):2554-2560.

7. Wang CY, Lai CC, Yang WC, et al. The association between inhaled corticosteroid and pneumonia in COPD patients: the improvement of patients' life quality with COPD in Taiwan (IMPACT) study. Int J Chron Obstruct Pulmon Dis. 2016;11:2775-2783. 
8. Cimen P, Unlu M, Kirakli C, et al. Should patients with COPD be vaccinated? Respir Care. 2015;60(2):239-243.

9. Kim J, Lee JK, Heo EY, Chung HS, Kim DK. The association of renin-angiotensin system blockades and pneumonia requiring admission in patients with COPD. Int J Chron Obstruct Pulmon Dis. 2016; 11:2159-2166.

10. Papp M, Li X, Zhuang J, Wang R, Uhal BD. Angiotensin receptor subtype AT(1) mediates alveolar epithelial cell apoptosis in response to ANG II. Am J Physiol Lung Cell Mol Physiol. 2002;282(4):L713-L718.

11. Mancini GB, Khalil N. Angiotensin II type 1 receptor blocker inhibits pulmonary injury. Clin Invest Med. 2005;28(3):118-126.

12. Liu CL, Shau WY, Chang CH, Wu CS, Lai MS. Pneumonia risk and use of angiotensin-converting enzyme inhibitors and angiotensin II receptor blockers. J Epidemiol. 2013;23(5):344-350.

13. Dial S, Nessim SJ, Kezouh A, Benisty J, Suissa S. Antihypertensive agents acting on the renin-angiotensin system and the risk of sepsis. Br J Clin Pharmacol. 2014;78(5):1151-1158.

14. Suberviola B, Rodrigo E, Gonzalez-Castro A, Serrano M, Heras M, Castellanos-Ortega Á. Association between exposure to angiotensinconverting enzyme inhibitors and angiotensin receptor blockers prior to septic shock and acute kidney injury. Med Intensiva. 2017;41(1): $21-27$.
15. Mortensen EM, Nakashima B, Cornell J, et al. Population-based study of statins, angiotensin II receptor blockers, and angiotensin-converting enzyme inhibitors on pneumonia-related outcomes. Clin Infect Dis. 2012;55(11):1466-1473.

16. Wu A, Good C, Downs JR, et al. The association of cardioprotective medications with pneumonia-related outcomes. PLoS One. 2014; 9(1):e85797.

17. Hsu WT, Lai CC, Wang YH, et al. Risk of pneumonia in patients with gastroesophageal reflux disease: a population-based cohort study. PLoS One. 2017;12(8):e0183808.

18. Otsuka M, Takahashi H, Shiratori M, Chiba H, Abe S. Reduction of bleomycin induced lung fibrosis by candesartan cilexetil, an angiotensin II type 1 receptor antagonist. Thorax. 2004;59(1):31-38.

19. Callera GE, Antunes TT, Correa JW, et al. Differential renal effects of candesartan at high and ultra-high doses in diabetic mice-potential role of the ACE2/AT2R/Mas axis. Biosci Rep. 2016;36(5):e00398.

20. Clancy P, Koblar SA, Golledge J. Angiotensin receptor 1 blockade reduces secretion of inflammation associated cytokines from cultured human carotid atheroma and vascular cells in association with reduced extracellular signal regulated kinase expression and activation. Atherosclerosis. 2014;236(1):108-115.

\section{Publish your work in this journal}

The International Journal of COPD is an international, peer-reviewed journal of therapeutics and pharmacology focusing on concise rapid reporting of clinical studies and reviews in COPD. Special focus is given to the pathophysiological processes underlying the disease, intervention programs, patient focused education, and self management protocols.

\section{Dovepress}

This journal is indexed on PubMed Central, MedLine and CAS. The manuscript management system is completely online and includes a very quick and fair peer-review system, which is all easy to use. Visit http://www.dovepress.com/testimonials.php to read real quotes from published authors. 